\title{
Aerobic bacterial Profile of Diabetic Foot Ulcers and their Antibiotic Sensitivity Pattern
}

\author{
Sharvari A. Samant*, Sharon Victor and Sandip Rai \\ ${ }^{1}$ Department of Microbiology, MGM Medical College and Hospital, Sector 18, Kamothe, \\ Navi Mumbai, Maharashtra-410209, India \\ ${ }^{2}$ Medical Microbiology, MGM Medical College and Hospital, Navi Mumbai, India \\ ${ }^{3}$ Department of Medicine, MGM Medical College and Hospital, Navi Mumbai, India
}

*Corresponding author

\begin{tabular}{|c|c|}
\hline & B S T R A C T \\
\hline Keywords & \multirow{3}{*}{$\begin{array}{l}\text { Diabetes mellitus is a global epidemic and diabetic foot ulcer (DFU) is one of its serious } \\
\text { complications. Diabetic foot infections are mixed bacterial infections and proper } \\
\text { management of these infections requires an appropriate antibiotic selection based on the } \\
\text { isolated organism. Type } 2 \text { diabetic patients affected with DFU and examined under } \\
\text { Wagner's classification 1-5 were included in the study. Two swabs were collected from } \\
\text { diabetic foot ulcer of each patient and the aerobic bacteria were identified and their } \\
\text { antibiogram was studied. It was found that } 80.70 \% \text { of the wounds were monobacterial and } \\
19.30 \% \text { were polybacterial in nature. Gram negative bacteria were predominant than Gram } \\
\text { Positive ones. Based on our antibiotic sensitivity results Aminoglycosides and } \\
\text { Fluroquinolones were more effective against Gram Negative bacteria and Tetracyclines } \\
\text { and Oxazolidinone against Gram Positive Bacteria. The authors suggest prompt initiation } \\
\text { of antibiotic therapy thereby reducing the burden of diabetic foot infections and preventing } \\
\text { morbidity. }\end{array}$} \\
\hline Article Info & \\
\hline $\begin{array}{l}7 \\
:\end{array}$ & \\
\hline
\end{tabular}

\section{Introduction}

Diabetes mellitus (DM) is a global epidemic and diabetic foot ulcer (DFU) is one of its serious complication.

The Indian diabetic population is expected to increase to 57 million by the year 2025. DFU results from a complex interaction of a number of risk factors. Individuals who develop DFU are at a greater risk of premature death, myocardial infarction and a fatal stroke than those without DFU.
Once the protective layer of skin is broken, deep tissues are exposed to bacterial infections that progress rapidly. This condition is characterized by several pathological complications such as neuropathy, peripheral vascular disease, foot ulceration and infection with or without oesteomyelitis which leads to development of gangrene and even necessitates limb amputation. Impaired metabolic mechanism leads to increased risk of infection and poor wound healing due to a series of mechanisms including diminished peripheral blood flow and decreased local 
angiogenesis. It has been reported that the individual with diabetes have at least a 10-fold greater risk of being hospitalized for soft tissue and bone infections of the foot than individuals without diabetes (Shakil and Khan, 2010).

In the diabetic patients, the microvascular circulation is affected which restricts the access of phagocytes and favors the infection. The local injuries and improper footwear further contribute to decrease in the blood supply in the lower extremities.

Most DFUs are polymicrobial in nature, with Gram positive cocci especially Staphylococci being the most common causative organism. Aerobic Gram negative bacilli are frequently isolated from infections that are chronic or follow antibiotic treatment and obligate anaerobes may be the co-pathogens associated with ischemic or necrotic wounds.

Most of the diabetic foot infections are polymicrobial in nature and proper management of these infections requires an appropriate antibiotic selection based on the isolated culture. Though empiric antibiotic regimen is based on available clinical and epidemiological data, definitive therapy should be based on culture of infected tissue. In this view a prospective research was planned to study the relative frequency of aerobic bacteria in diabetic foot and their antibiotic sensitivity pattern.

\section{Materials and Methods}

This study was carried out in a period of one year in Department of Microbiology, MGM Medical College and Hospital, Navi Mumbai. Type 2 diabetic patients affected with DFU and examined under Wagner's classification 1-5 were included in the study. Two swabs were collected from the foot ulcers of each of the 30 diabetic patients.
Out of the two swabs collected, one was used for preparation of smears. The smears were Gram stained and observed for presence of pus cells, epithelial cells and bacteria.

The other swab was inoculated on Blood agar, MacConkey's agar and Mannitol Salt Agar. The organisms were identified upto the species level by standard microbiological methods (Mackie and McCartney, 2011) and antibiotic sensitivity was carried out using Kirby bauer's Disc Diffusion Method (1966) and the results were interpreted as per CLSI guidelines (2012).

The following antibiotics were tested against Gram Negative and Gram Positive bacteria.

Gram Negative bacteria: Gentamicin (GEN), Ciprofloxacin (CIP), Ofloxacin (OF), Amikasin (AK), Tobramycin (TOB), Cefoparazone (CPZ), Augmentin (AMC), Ceftazidime (CAZ), Cefotaxime (CTX), Tetracycline (TE), Cefuroxime (CXM), Cefazolin $(\mathrm{CZ})$

Gram Positive Bacteria: Gentamicin (GEN), Ciprofloxacin (CIP), Linezolid (LZ), Clindamycin (CD), Roxithromycin (RO), Azethromycin (AZM), Cefoxitin (CX), Augmentin (AMC), Tetracycline (TE), Cefuroxime (CXM), Cefazolin (CZ), Cotrimoxazole (COT)

\section{Results and Discussion}

Microbiological analysis of total of 30 patients with Diabetic Foot ulcers was done. The results are as under, Diabetic foot ulcers are a common and serious complication of chronic DM which often develop because of uncontrolled diabetes and incomplete selfcare. Although there are numerous studies with regard to the clinical profile and microbiological analysis, the magnitude of the problem is just the same as earlier. 
In this prospective study of 30 patients of diabetic foot ulcers, it was observed that even though the age group varied between 40-75 years, the mean age group was 54 years and Male to Female ratio was 2:1. This is in agreement with Manisha Jain et al., (2012). It can be because men have nearly twice the odds of having sensory neuropathy as women which is attributed to the longer nerve length in men.

In this study, $87 \%$ samples showed aerobic growth out of which nearly $80.70 \%$ infections were monobacterial. Lower incidence of polymicrobial growth $(19.30 \%)$ may be due to prior antibiotic treatment. Monobacterial infections are easy to manage as compared to polybacterial infections. Polybacterial infections are difficult to cure because these bacteria form biofilms and impede the activity of antibiotics (Cintron et al., 2012). Furthermore the interaction among the organisms leads to release of virulence factors and agents which increase inflammation and act synergistically to cause a chronic wound infection (Lipsky et al., 2013).

The swabs when cultured grew variety of Gram Positive and Gram negative organisms. Staphylococcus aureus (75\%) and Enterococcus spp. (25\%) were the most frequent isolated Gram Positive bacteria. Pseudomonas aeruginosa (34.7\%) was the most predominant GNB followed by E. coli (30.4\%), Proteus vulgaris and Enterobacter (8.69\%) etc. Almost similar results have been reported by Bansal et al., (2008) and Shankar et al., (2005). The difference observed in the prevalence of GNBs in DFI between diabetic patients of Eastern and Western countries remain largely unknown. However the environmental factors such as sanitary habits like use of water for peri anal wash after defecation leads to contamination of hands.

Fig.1 Age group of patients with DFU

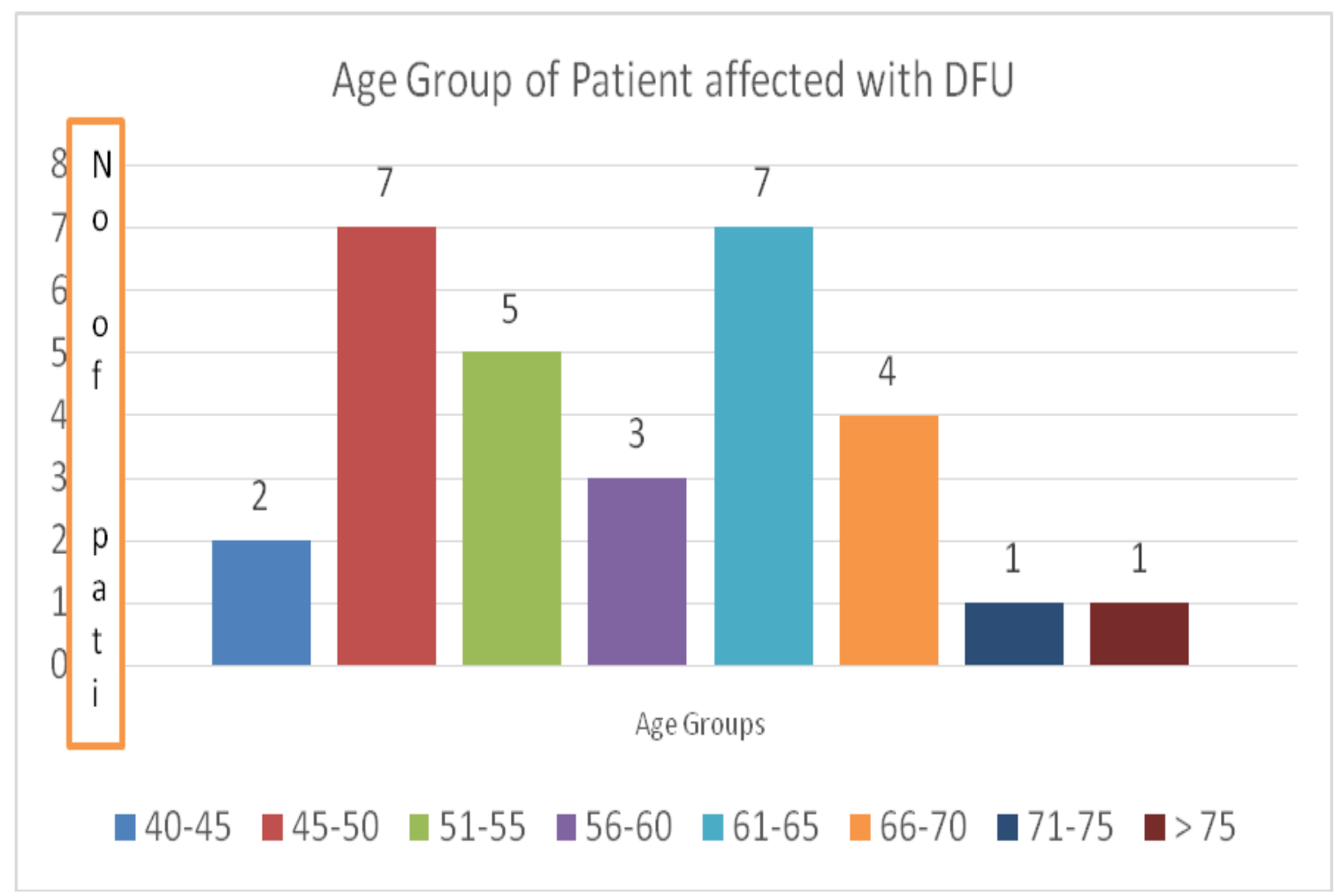


ig.2 Sex distribution of patients with DFU

\section{Sex Distribution of Patients with DFU}

7

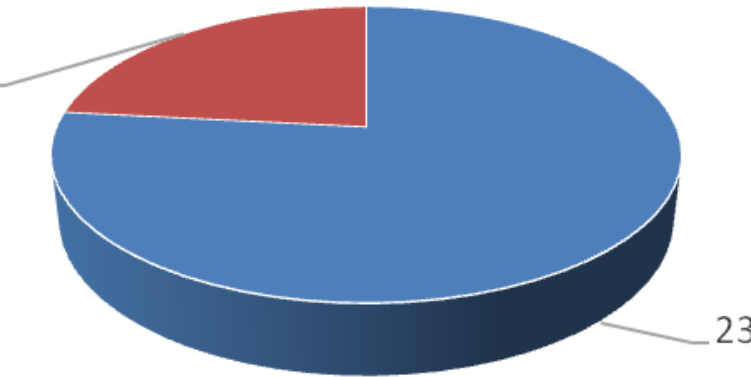

- Males - Females

Fig.3 Pie chart of DFU patients showing monobacterial and polybacterial growth

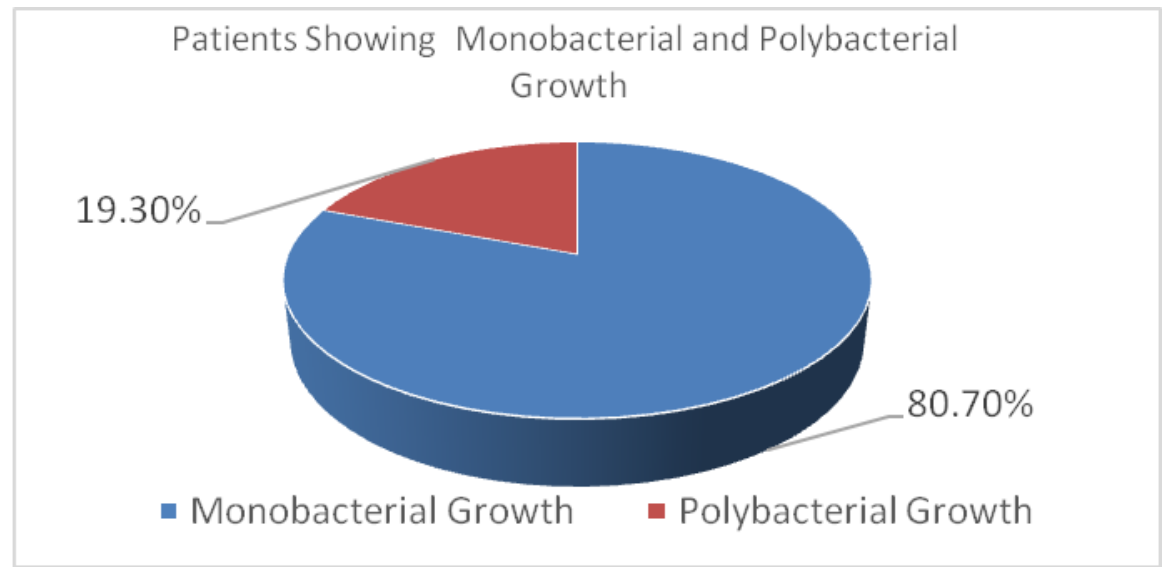

Fig.4 Spectrum of Gram positive organisms in DFU

\section{Spectrum of Gram Positive Organisms in DFU} $25 \%$

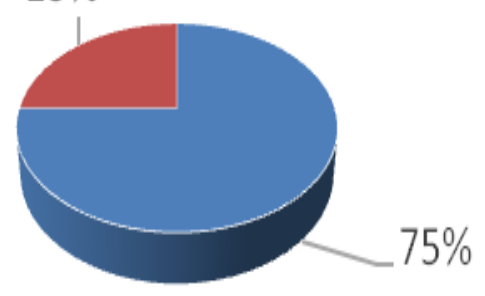

- Staphylococcus aureus - Enterococcus Spp. 
Fig.5 Spectrum of Gram negative bacteria in DFU

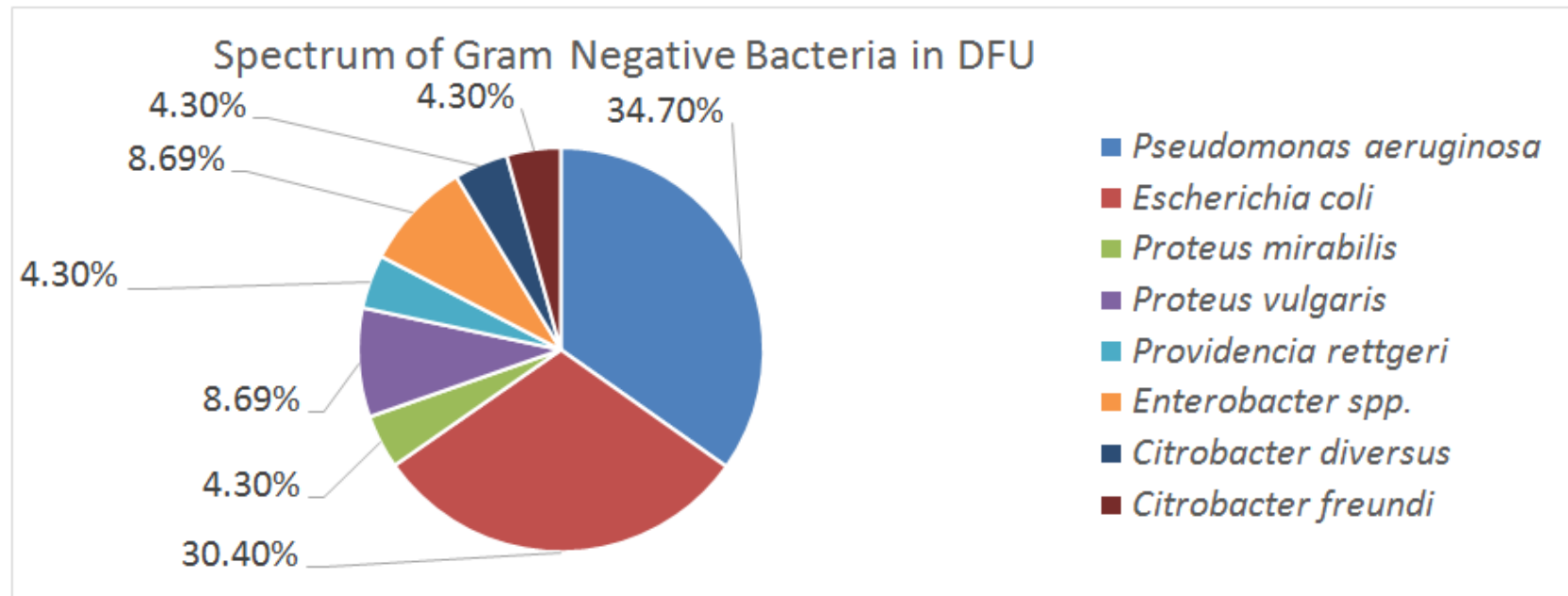

Fig.6 Antibiotic sensitivity pattern of common GNBs in DFU
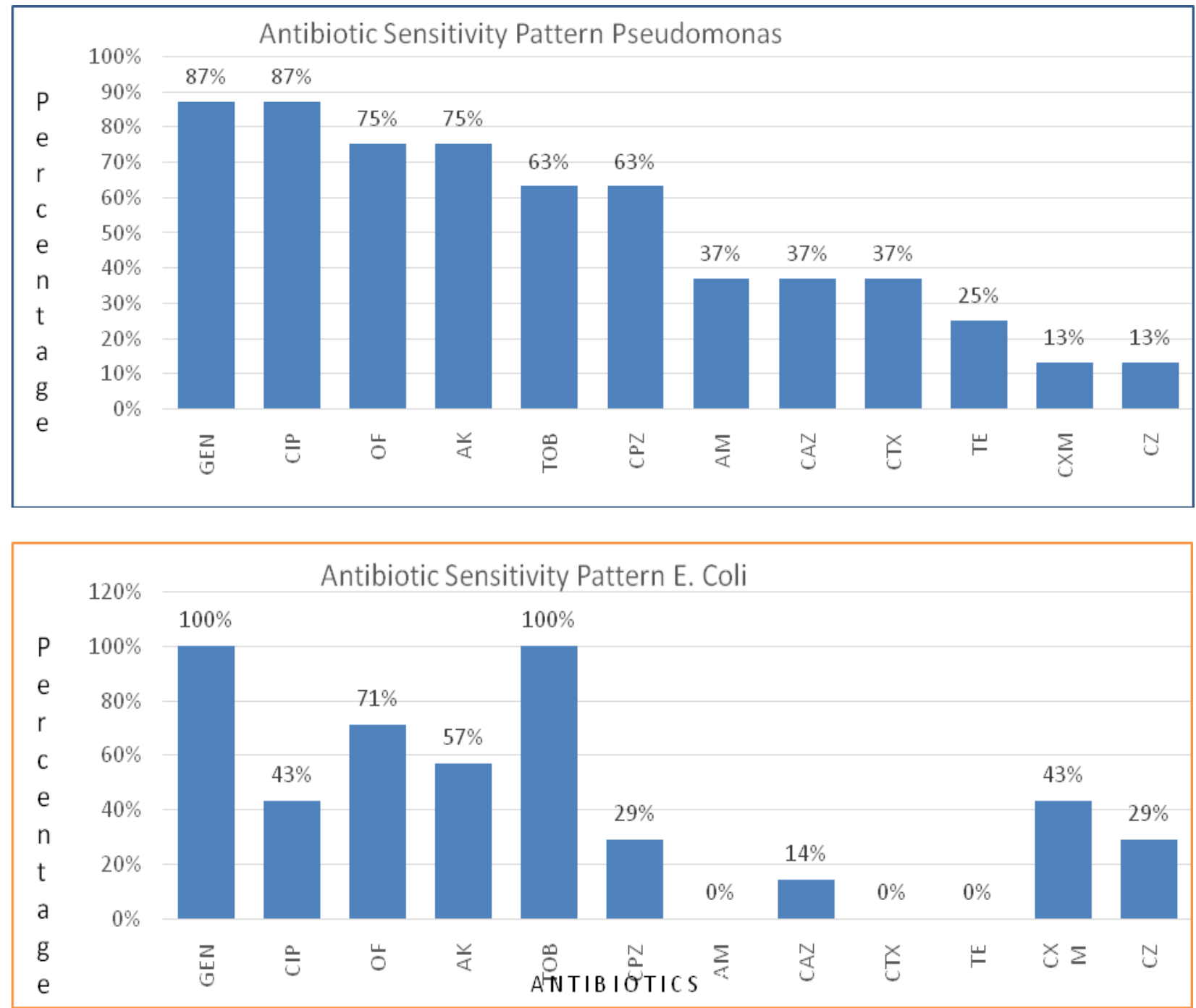
Fig.7 Antibiotic sensitivity pattern of Gram positive bacteria (Staphylococcus aureus $\mathrm{N}=6$ )

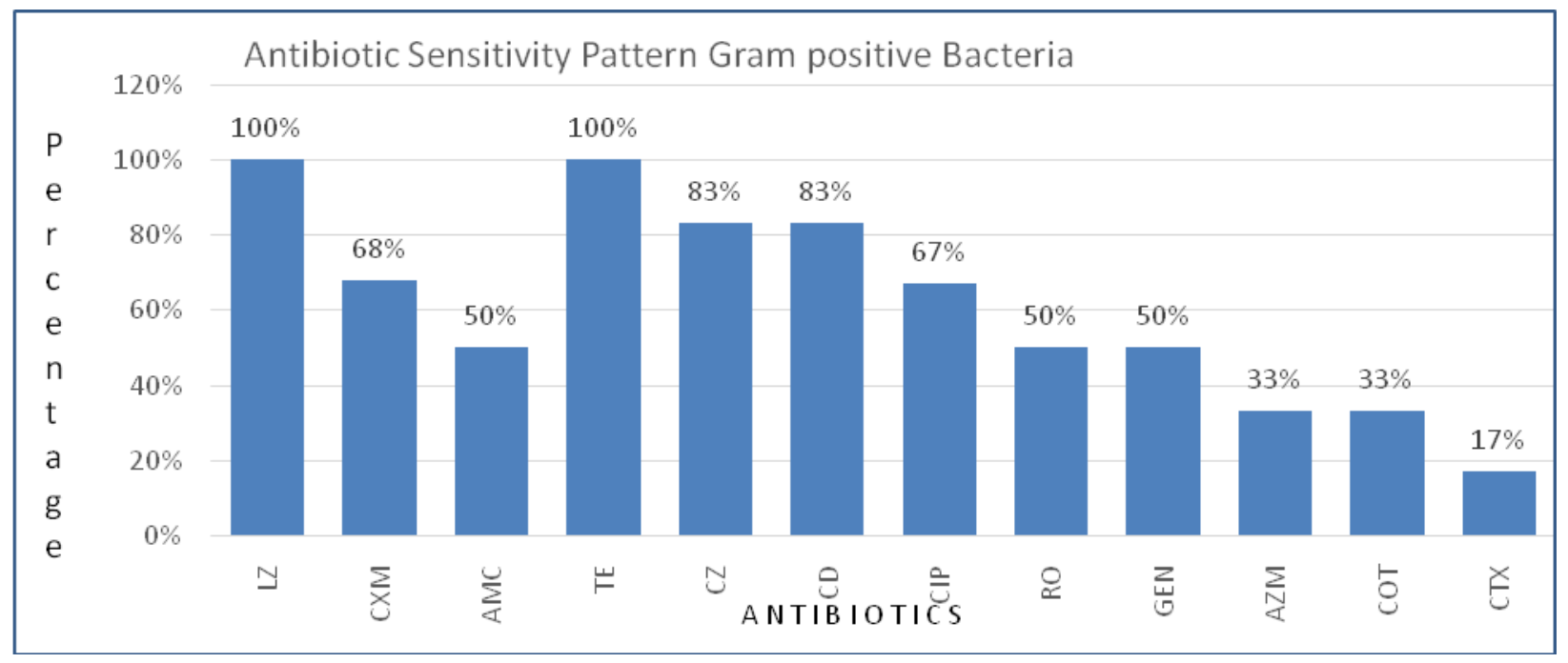

All the isolates were subjected to antibiotic sensitivity test and the sensitivity pattern was noted.

Amongst the Gram Positive organisms obtained in culture, all the isolates of Staphylococcus aureus were found sensitive to Tetracycline and Linezolid and Vancomycin and 50\% isolates were sensitive to Augmentin, Co-Trimoxazole and Gentamicin. This finding is similar to M. Jain et al., (2012) who reported 90\% sensitivity to Linezolid and Patil et al., (2017) who reported $92.45 \%$ sensitivity to Vancomycin.

Amongst the Gram Negative bacteria, Pseudomonas aeruginosa was the commonest organism and moderate to high level sensitivity was observed against Gentamicin (87\%), Ciprofloxacin (87\%), Ofloxacin (75\%) and Amikacin (75\%) followed by Tobramycin (63\%). E. coli showed $100 \%$ sensitivity to Tobramycin and Gentamicin and 100\% resistance to Cefuroxime.

Based on our results of antimicrobial susceptibility results, it was observed that Aminoglycosides and fluoroquinolones were found to be effective against Gram negative bacteria and Tetracyclines and Oxazolidinone were found to be effective against Gram positive organisms (Fig. 1-7).

Diabetic foot ulcers the most common complication of diabetes mellitus develops as a result of peripheral neuropathy or ischemia or both and may lead to limb amputation or life threatening condition. This can lead to hospitalization, morbidity and mortality. Prompt initiation of antibiotic therapy as well as surgical debridement of necrotic of devascularized soft tissue and are essential for controlling the infection and preventing additional morbidity.

Appropriate usage of antibiotics based on local antibiogram pattern can certainly help the clinicians in reducing the burden of diabetic foot infections, which ultimately reduce the rate of amputations.

\section{References}

Bansal E, Garg A, Bhatia S, Attri, AK, Chandar J. 2008. Spectrum of Microbial Flora in Diabetic Foot Ulcers. 51(2): 204-208.

Bauer AW, Kirby WMM, Sherris JC, Turch 
M. 1966. Antibiotic Susceptibility Testing by a Standardized Single Disc Method. AMJ by ClinPatho. 36; 493496.

Citron DM, Goldstein EJ, Merriam CV, Lipsky BA, Abramson MA. 2007. Bacteriology of Moderate to Severe Diabetic Foot Infections and in vitro Activity of Antimicrobial Agents. J ClinMicrobiol. 45: 2819-28.

CLSI. 2012. Performance Standards for Antimicrobial Susceptibility Testing. 11th Edition. 32(1).

Lipsky BA, Richard JL, Lavigne JP. 2013. Diabetic Foot Ulcer Microbiome; One small Step for Molecular Microbiology. One Giant Leap for Understanding Diabetic Foot Ulcers. Diabetes. 62: 67981.

Mackie and McCartney Practical Microbiology 2011. $14^{\text {th }}$ Edition. Churchill Livingston Press.

Manisha Jain, Patel Mitesh, Sood Nidhi, Modi
Dhara, Vegad M. 2012. Spectrum of Microbial Flora in Diabetic Foot Ulcer and its Antibiotic Sensitivity Pattern in Tertiary Care Hospital in Ahmedabad, Gujarat. National Journal of Medical Research. 2(3): 354-357.

Patil S., Mane R., Bacterial and Clinical Profile of DFU using Optimal Culture Techniques. 2017. International Journal of Research in Medical Sciences. 5(2): 496-502.

Shakil S., Khan AU. 2010. Infected foot ulcers in Male and Female Diabetic Patients. A Clinico bio-informative study. Ann Clinical Microbiology. 9: 110.

Shankar EM, Mohan V, Premlatha G, Shriniwasan RS, Usha AR. 2005. Bacteriological Etiology of Diabetic Foot Infections in South India. Eur J Intern Med. 16: 567-570.

\section{How to cite this article:}

Sharvari A. Samant, Sharon Victor and Sandip Rai. 2018. Aerobic bacterial Profile of Diabetic Foot Ulcers and their Antibiotic Sensitivity Pattern. Int.J.Curr.Microbiol.App.Sci. 7(01): 14121418. doi: https://doi.org/10.20546/ijcmas.2018.701.173 